\title{
First Price Auctions: Monotonicity and Uniqueness*
}

\author{
Gustavo E. Rodriguez
}

February 1997

\begin{abstract}
I study monotonicity and uniqueness of the equilibrium strategies in a two-person first price auction with affiliated signals. I show that when the game is symmetric there is a unique Nash equilibrium that satisfies a regularity condition requiring that the equilibrium strategies be piecewise monotone. Moreover, when the signals are discrete-valued, the equilibrium is unique. The central part of the proof consists of showing that at any regular equilibrium the bidders' strategies must be monotone increasing within the support of winning bids. The monotonicity result derived in this paper provides the missing link for the analysis of uniqueness in twoperson first price auctions. Importantly, this result extends to asymmetric auctions.
\end{abstract}

\section{Introduction}

Much of the literature on auctions focuses on symmetric equilibria in which the buyers' bids increase monotonically in the signals they observe. For symmetric first price auctions there is only one symmetric equilibrium in monotone strategies, but it is not yet fully understood whether there may be other equilibria that do not satisfy symmetry and monotonicity. There are a few authors who address the question of uniqueness but only in very specific environments. For instance, Griesmer, Levitan and Shubik [2] provide a complete characterization of the equilibrium set of a two-person first price auction in which the bidders' private valuations are independently and uniformly distributed. More recently, Maskin and Riley [9] establish uniqueness for the independent private values model with symmetric bidders and provide partial extensions for asymmetric versions of that model. However, a general treatment of environments that involve common values and correlated signals is still missing. Maskin and Riley [7] constitutes a first

${ }^{*}$ This paper is part of the second chapter of my Ph.D thesis. 
step in tackling this problem. For the case of a two-person symmetric auction with affiliated signals, that paper shows that the symmetric equilibrium is the only equilibrium in monotone strategies.

Ruling out the possibility that there are other equilibria in non-monotone strategies remains as the main hurdle to solving the problem of uniqueness in the two-person case. I do this here. Specifically, I examine this problem in the context of a sealed bid first price auction with affiliated signals. Aside from the restriction to two players, the environment that I consider is rather general, encompassing the general symmetric model of Milgrom and Weber [12] as well as the model of Maskin and Riley [7] as particular cases.

The main findings are the following. I show that at any Nash equilibrium that satisfies a regularity condition, both bidders' strategies must actually be monotone increasing within the support of bids that can win with positive probability (winning bids). The regularity condition requires that the equilibrium strategies employed by the bidders be piecewise monotone. Using this result, it then follows that there is a unique equilibrium when the game is symmetric. Moreover, when signals are discrete-valued every equilibrium is regular so there must be a unique equilibrium in this case as well.

Most of the paper is devoted to show that the equilibrium strategies must be monotone increasing. Importantly, this result extends to asymmetric auctions. The argument is rather elaborate and relies heavily on the assumption of affiliation. I briefly sketch the main issues involved.

To examine the problem of bidder 1 , fix bidder 2's strategy and two bids $b_{0}$ and $b_{1}$, with $b_{0}<b_{1}$. Let $\pi_{1}\left(b, s_{1}\right)$ denote his expected payoff if he bids $b$ and his signal is $s_{1}$. Moreover, let $\delta_{1}\left(s_{1}, s_{2}\right)$ denote the increase in his expected payoff due to an increase in his bid from $b_{0}$ to $b_{1}$, given that the signals are $s_{1}$ and $s_{2}$. Also define a function: $h_{1}(x, y)=E\left(\delta_{1}\left(x, \mathbf{s}_{2}\right) \mid \mathbf{s}_{1}=y\right)$ and notice that $\pi_{1}\left(b_{1}, s_{1}\right)-\pi_{1}\left(b_{0}, s_{1}\right)=h_{1}\left(s_{1}, s_{1}\right)$.

To study the best reply of bidder 1 to the strategy $\beta_{2}$ of bidder 2 , we need to gain insight about the way $\pi_{1}\left(b_{1}, s_{1}\right)-\pi_{1}\left(b_{0}, s_{1}\right)$ varies with $s_{1}$. The two arguments of $h_{1}$ reflect two different aspects of the information revealed by the signal $s_{1}$. The first argument reflects direct information about the expected value of the object. Given the monotonic structure introduced in the problem by the assumption of affiliation, this expected value increases with $s_{1}$. Thus, $\delta_{1}$ is nondecreasing in $s_{1}$ and, therefore, $h_{1}$ is non-decreasing in its first argument. The second argument of $h_{1}$ reflects information about both the expected value of the object and player 2's bidding behavior, which is revealed by $s_{1}$ indirectly through its statistical linkage with $s_{2}$.

To understand this second effect I rely on the following property of affiliated random variables. I say that the map $\varphi: \Re \rightarrow \Re$ is quasi sign-monotone (QSM) if $\varphi(y) \geq 0 \Rightarrow \varphi\left(y^{\prime}\right) \geq 0$ for almost every $y^{\prime}>y$. The map $\varphi$ is weakly $Q S M$ if $\varphi(y)>0 \Rightarrow \varphi\left(y^{\prime}\right) \geq 0$ for almost every $y^{\prime}>y$. Suppose that $\mathbf{x}$ and $\mathbf{y}$ are affiliated random variables. Then, if $\varphi$ is weakly QSM, $E(\varphi(\mathbf{y}) \mid \mathbf{x}=x)$ is QSM. 
Let $\underline{b}$ denote the upper lower bound of the support of winning bids. A key property is that $\delta_{1}$ is QSM in its second argument as long as the restriction of the inverse bidding strategy to $[\underline{b}, b]$ is monotone increasing. Combining these observations we are able to conclude that $h_{1}$ is QSM in its second argument. Taking into account both arguments of $h_{1}$, we reach the conclusion that if the restriction of the inverse bidding strategy to $\left(\underline{b}, b_{0}\right]$ is monotone increasing, then $\pi_{1}\left(b_{1}, s_{1}^{\prime}\right)-\pi_{1}\left(b_{0}, s_{1}^{\prime}\right) \geq 0 \Rightarrow \pi_{1}\left(b_{1}, s_{1}\right)-\pi_{1}\left(b_{0}, s_{1}\right)>0$ for $s_{1}>s_{1}^{\prime}$.

This property plays a central role in the monotonicity argument. As an example, suppose that both buyers' inverse bidding strategies are monotone increasing on $[\underline{b}, b]$, but bidder 1's strategy is not monotone outside this interval: he bids $b^{\prime}>b$ when his signal is $s_{1}^{\prime}<s_{1}$ and bids $b$ when his signal is $s_{1}$. The optimality $b^{\prime}$ for $s_{1}^{\prime}$ implies that $\pi_{1}\left(b^{\prime}, s_{1}^{\prime}\right)-\pi_{1}\left(b, s_{1}^{\prime}\right) \geq 0$. Consequently, the preceding property implies that $\pi_{1}\left(b^{\prime}, s_{1}\right)-\pi_{1}\left(b, s_{1}\right)>0$, which contradicts the optimality of $b$ for $s_{1}$. In fact, this kind of reasoning allows us to rule out all non-monotonic behavior as long as the bidders use piecewice monotone strategies in equilibrium.

The paper is organized as follows. In section 2 I lay out the model and introduce the basic notation. In section $3 \mathrm{I}$ derive some basic results about the support of the equilibrium distribution of bids. In section 4 I derive the key consequences of the assumption that the signals are affiliated. Section 4 contains the main monotonicity result and section 5 applies this result to establish uniqueness in the symmetric case. Section 6 is devoted to concluding remarks. Most of the proofs are contained in the appendix.

\section{The Model}

\subsection{Description and Basic Assumptions}

In this section I lay out the model of a sealed bid first price auction. There are two bidders who submit their bids simultaneously and a single object is awarded to the highest bidder as long as his bid is at least as large as a reserve price $r$. In case of a tie, the winner is selected randomly. Moreover, the price paid by the winner equals his own bid.

The problem is modeled as a two-person Bayesian game. Throughout the paper I refer to the players as bidder $i$ or bidder $j$, making the convention that $i, j=1,2$ and that $i \neq j$. Each player selects his bid after privately observing the value of a signal. Let $\mathbf{s}_{i}$ denote the signal observed by bidder $i$ (where I adopt the convention of using bold case letters for random variables). This signal takes values in a set $S_{i} \subset \Re$, which I suppose to be either a closed interval or a finite set. Let $F: S_{1} \times S_{2} \rightarrow[0,1]$ denote the joint distribution of the players' signals. I suppose that $F$ has a density function $f$ with support $S_{1} \times S_{2}$ (in the discrete case, $f$ denotes the density with respect to the counting measure). Moreover, I assume that the signals are affliated. In general, the components of a random 
vector are affiliated if any two non-decreasing functions of the random vector are positively correlated conditional on every sublattice of the support of the joint distribution. In particular, random variables whose distribution has a density are affiliated if and only if the probability density function is Monotone Totally Positive of Order 2 ( $\left.M T P_{2}\right)$ (Karlin and Rinott [5], Milgrom and Weber [12]). In the present case, this means that the distribution of signals satisfies the following assumption:

$\left(A_{1}\right) \quad f(s) f\left(s^{\prime}\right) \leq f\left(s \vee s^{\prime}\right) f\left(s \wedge s^{\prime}\right)$ for every $s, s^{\prime} \in S_{1} \times S_{2}$, where $s \vee s^{\prime}$ and $s \wedge s^{\prime}$ respectively denote the componentwise maximum and minimum of $s$ and $s^{\prime}$.

The bidders' preferences are represented by a von Neumann-Morgenstern utility function. For each $\left(s_{1}, s_{2}\right) \in S_{1} \times S_{2}$, let $u_{i}\left(b, s_{i}, s_{j}\right)$ denote bidder $i$ 's utility if he makes a bid $b \in \Re_{+}$and obtains the object. The utility function is normalized so that the utility of a bidder that does not obtain the object is zero. I also assume that $u_{i}$ satisfies the following conditions:

$\left(A_{2}\right)$ The function $u_{i}$ is twice continuously differentiable, strictly decreasing in $b$, strictly increasing in $s_{i}$ and non-decreasing in $s_{j}$.

$\left(A_{2}\right) \quad$ For $b_{1}>b_{0}$, the difference $u_{i}\left(b_{1}, s_{i}, s_{j}\right)-u_{i}\left(b_{0}, s_{i}, s_{j}\right)$ is non-decreasing in $s_{i}$.

\subsection{Additional Notation}

Here I define the players' strategies and several concepts of monotonicity and local monotonicity that will be useful in the analysis. Moreover, I introduce additional notation that will be used throughout the paper.

Let $\mathcal{B}$ denote the Borel $\sigma$-algebra associated to $\Re_{+}$and let $\mathcal{P}(\mathcal{B})$ denote the set of probability measures on $\mathcal{B}$. A behavior strategy for bidder $i$ is a mapping $\beta_{i}$ : $S_{i} \rightarrow \mathcal{P}(\mathcal{B})$ such that, for each $B \in \mathcal{B}$, the application $\left\langle\beta_{i}, B>\right.$ is a measurable function from $S_{i}$ to the interval $[0,1]$. The mapping $\tilde{\beta}_{i}: S_{i} \rightarrow \Re$ is a selection from $\beta_{i}$ if $\tilde{\beta}_{i}\left(s_{i}\right) \in \operatorname{supp} \beta_{i}\left(s_{i}\right)$ for every $s_{i} \in S_{i}$. I also denote $\beta_{i}^{-1}(b)=\left\{s_{i} \in\right.$ $\left.S_{i}: b \in \operatorname{supp} \beta_{i}\left(s_{i}\right)\right\}$. The strategy $\beta_{i}$ and the marginal distribution of bidder $i$ 's signal induce a probability distribution over buyer $i$ 's bids. The infimum and the supremum of the support of this distribution will be denoted $\underline{b}_{i}$ and $\bar{b}_{i}$, respectively. Given a strategy profile $\beta=\left(\beta_{1}, \beta_{2}\right)$, I will denote $\underline{b}=\max \left\{\underline{b}_{1}, \underline{b}_{2}, r\right\}$ and $\bar{b}=\max \left\{\bar{b}_{1}, \bar{b}_{2}\right\}$.

The following concepts of monotonicity and sign-monotonicity of a function provide a convenient way of stating the results of this paper. Let $Y$ denote a measurable subset of $\Re$ and consider a mapping $\varphi: Y \rightarrow \Re$. I say that $\varphi$ is monotone non-decreasing (increasing) if $\varphi(y) \leq(<) \varphi\left(y^{\prime}\right)$ when $y<y^{\prime}$. I say that $\varphi$ is quasi sign-monotone $(Q S M)$ if $\varphi(y) \geq 0 \Rightarrow \varphi\left(y^{\prime}\right) \geq 0$ for almost every $y^{\prime}>y$. 
The map $\varphi$ is weakly $Q S M$ if $\varphi(y)>0 \Rightarrow \varphi\left(y^{\prime}\right) \geq 0$ for almost every $y^{\prime}>y$. The map $\varphi$ is sign-monotone (SM) if it is QSM and $\varphi(y)>0 \Rightarrow \varphi\left(y^{\prime}\right)>0$ for almost every $y^{\prime}>y$. Finally, $\varphi$ is strictly sign-monotone $(S S M)$ if $\varphi(y) \geq 0 \Rightarrow \varphi\left(y^{\prime}\right)>0$ for almost every $y^{\prime}>y$.

Applying the preceding definitions to our strategy space, I define the following concepts. I say that the strategy $\beta_{i}$ is monotone non-decreasing (increasing) if the set of the selections $\tilde{\beta}_{i}$ from $\beta_{i}$ such that $\tilde{\beta}_{i}$ is not monotone non-decreasing (increasing) is a set of probability measure zero (according to the probability measure induced by $\beta_{i}$ ). Moreover, I say that $\beta_{i}$ is monotone at $b$ (quasi-monotone $a t b$ ) if the set of selections $\tilde{\beta}_{i}$ from $\beta_{i}$ such that $\tilde{\beta}_{i}-b$ is not SM (QSM) at $b$ is a set of probability measure zero.

The following notation will be used throughout the paper. Suppose that bidder $j$ employs the strategy $\beta_{j}$. Given $\beta_{j}$, let $Q_{i}\left(b, s_{j}\right)$ denote the probability that bidder $i$ obtains the object provided that he bids $b$ and that bidder $j$ 's signal is $s_{j}$. Also let $\pi_{i}\left(b, s_{i}\right)$ denote bidder $i$ 's expected payoff when he bids $b$ and his signal is $s_{i}$. More explicitly, $\pi_{i}\left(b, s_{i}\right)=E\left(u_{i}\left(b, \mathbf{s}_{i}, \mathbf{s}_{j}\right) Q_{i}\left(b, \mathbf{s}_{j}\right) \mid \mathbf{s}_{i}=x\right)$. Since $Q_{i}\left(b, s_{j}\right)$ is monotone non-decreasing in its first argument, the corresponding right and left limits exist everywhere. I denote $Q_{i}\left(b^{+}, s_{j}\right)=\lim _{t \downarrow b} Q_{i}\left(t, s_{j}\right)$ and $Q_{i}\left(b^{-}, s_{j}\right)=\lim _{t \uparrow b} Q_{i}\left(t, s_{j}\right)$. Similarly, I denote $\pi_{i}\left(b^{+}, s_{i}\right)=\lim _{t \downarrow b} \pi_{i}\left(t, s_{i}\right)$ and $\pi_{i}\left(b^{-}, s_{i}\right)=\lim _{t \uparrow b} \pi_{i}\left(t, s_{i}\right)$. The following result follows directly from the definitions.

Lemma 1 Suppose that $\beta_{j}$ is monotone at $b$. Then there is a parameter $\hat{s}_{j}$ such that $Q_{i}\left(b^{+}, s_{j}\right)=1$ for almost every $s_{j}<\hat{s}_{j}$ and $Q_{i}\left(b^{+}, s_{j}\right)=0$ for almost every $s_{j}>\hat{s}_{j}$.

\section{Preliminary Results about the Equilibrium Bidding Distributions}

This section reviews some properties of the support of the equilibrium bidding distributions that do not directly depend on the assumption that the signals are affiliated. In essence, I rule out the possibility of gaps and, in some sense, mass points in the interior of the support of winning bids. Since most of the arguments are already standard, I will only discuss the results briefly. Nonetheless, detailed proofs are supplied in the appendix for completeness.

The first observation is that none of the bidders ever selects a winning bid at which his expected payoff exhibits a jump discontinuity. Since this kind of discontinuity can only occur because the other bidder's strategy has a mass point, the tie-breaker makes the expected payoff of the player in question an average of the right and left limits of his expected payoff at the point of discontinuity. But then he can improve by either bidding a little more or a little less. Thus, we can state the following: 
Lemma 2 Suppose that the bid $\hat{b}>\underline{b}$ is optimal for bidder $i$ when he observes the signal $x$. Then $\pi_{i}(b, x)$ is continuous at $\hat{b}$.

To rule out gaps in the support the intuition is simple: If a bidder's support presents a gap $(b-\varepsilon, b]$, the other bidder never bids an amount close to $b$, since he can reduce his bid, and expected payment, without affecting his chance of winning. This line of thought leads to contradiction, establishing the following result.

Lemma 3 The support of the distribution of bids of each bidder contains the interval $[\underline{b}, \bar{b}]$. Moreover, $\bar{b}_{1}=\bar{b}_{2}=\bar{b}$.

Finally, note that when a bidder's strategy is quasi-monotone and presents a mass point at a bid $b$, the "types" of this player that bid $b$ are larger than the ones that bid less than $b$. Since the other bidder can increase his payoff by increasing slightly his bid to "capture" these higher types, his payoff jumps discontinuously at $b$. Thus, he never bids in some interval $(b-\varepsilon, b]$, contradicting Lemma 3. In fact, one can show the following.

Lemma 4 Suppose that $\beta_{j}$ is quasi-monotone at $b \in(\underline{b}, \bar{b}]$. Then $\beta_{j}$ is atomless and, consequently, monotone at $b$.

\section{Consequences of Affiliation}

Given the strategy of the other bidder, the effect on a bidder's expected payoff of a change in his bid only depends on the value of his signal. Clearly, the nature of this dependence is critical in the analysis of the shape of the equilibrium strategies that we undertake in the next section. Here I investigate how that dependence is affected by the assumption that the signals are affiliated.

For the remainder of this section I fix the strategy $\beta_{j}$ of bidder $j$ in order to examine the best response by bidder $i$. I also fix two bids $b_{0}$ and $b_{1}$, where $b_{0}<b_{1}$. To examine the dependence of the difference $\pi_{i}\left(b_{1}, s_{i}\right)-\pi_{i}\left(b_{0}, s_{i}\right)$ on the signal $s_{i}$, I introduce the following concepts which will help articulate the analysis. I define a mapping $\delta_{i}: S_{i \times} S_{j} \rightarrow \Re$ such that $\delta_{i}\left(s_{i}, s_{j}\right)=u_{i}\left(b_{1}, s_{i}, s_{j}\right) Q_{i}\left(b_{1}, s_{j}\right)-$ $u_{i}\left(b_{0}^{+}, s_{i}, s_{j}\right) Q_{i}\left(b_{0}^{+}, s_{j}\right)$. In words, $\delta_{i}\left(s_{i}, s_{j}\right)$ represents the increase in bidder $i$ 's expected payoff due to an increase in his bid from a bid just above $b_{0}$ to $b_{1}$, given that the bidders' signals take the values $s_{i}$ and $s_{j}$. I also define a mapping $h_{i}: S_{i} \times S_{j} \rightarrow \Re$ such that $h_{i}(x, y)=E\left(\delta_{i}\left(x, \mathbf{s}_{j}\right) \mid \mathbf{s}_{i}=y\right)$. Notice that $\pi_{i}\left(b_{1}, s_{i}\right)-$ $\pi_{i}\left(b_{0}^{+}, s_{i}\right)=h_{i}\left(s_{i}, s_{i}\right)$. Thus, the first argument of $h_{i}$ reflects the dependence of $\pi_{i}\left(b_{1}, s_{i}\right)-\pi_{i}\left(b_{0}^{+}, s_{i}\right)$ on $s_{i}$ through the direct dependence of $\delta_{i}$ on $s_{i}$ and the second argument reflects the indirect dependence on $s_{i}$ through the conditional distribution of $\mathbf{s}_{j}$. 
Naturally, a first step to understand $h_{i}$ relies on the analysis of $\delta_{i}$. Consider first the dependence of $\delta_{i}$ on $s_{i}$. Note that

$$
\begin{aligned}
& \delta_{i}\left(s_{i}^{\prime}, s_{j}\right)-\delta_{i}\left(s_{i}, s_{j}\right)= \\
& =Q_{i}\left(b_{1}, s_{j}\right)\left[u_{i}\left(b_{1}, s_{i}^{\prime}, s_{j}\right)-u_{i}\left(b_{1}, s_{i}, s_{j}\right)\right]-Q_{i}\left(b_{0}^{+}, s_{j}\right)\left[u_{i}\left(b_{0}, s_{i}^{\prime}, s_{j}\right)-u_{i}\left(b_{0}, s_{i}, s_{j}\right)\right] \\
& \geq\left[Q_{i}\left(b_{1}, s_{j}\right)-Q_{i}\left(b_{0}^{+}, s_{j}\right)\right]\left[u_{i}\left(b_{0}, s_{i}^{\prime}, s_{j}\right)-u_{i}\left(b_{0}, s_{i}, s_{j}\right)\right]
\end{aligned}
$$

where the inequality is a consequence of assumption $A_{3}$. Thus, assumption $A_{2}$ and the fact that $Q_{i}\left(b_{1}, s_{j}\right)-Q_{i}\left(b_{0}, s_{j}\right) \geq 0$ imply that $\delta_{i}$ is non-decreasing in its first argument, and that it is strictly increasing when the preceding inequality is strict. Thus, if bidder $i$ 's probability of obtaining the object is strictly larger when he bids $b_{1}$ than when he bids $b_{0}$, as it will be in the relevant cases, $h_{i}$ will be strictly increasing in the first argument.

Examination of the dependence of $\delta_{i}$ on $s_{2}$ is somewhat more intricate. Suppose that the restriction of bidder $j$ 's inverse bidding strategy to the interval $\left[\underline{b}_{j}, b_{0}\right)$ is monotone increasing. Since this implies that $\beta_{j}$ is monotone at $b_{0}$, Lemma 4 implies that it is also atomless at $b_{0}$. Clearly, there must exist a signal $\hat{s}_{j}$ such that if bidder $i$ bids $b_{0}$, he wins if and only if $s_{j}<\hat{s}_{j}$. Although bidder $i$ also wins with $b_{1}$, he prefers to win with a lower bid. Then, $\delta_{i}\left(s_{i}, s_{j}\right)=$ $u_{i}\left(b_{1}, s_{i}, s_{j}\right)-u_{i}\left(b_{0}, s_{i}, s_{j}\right)<0$ when $s_{j}<\hat{s}_{j}$. On the other hand, when $s_{j}>\hat{s}_{j}$, bidder $i$ loses when he bids $b_{0}$. Thus, in this case $\delta_{i}\left(s_{i}, s_{j}\right)=Q_{i}\left(b_{1}, s_{j}\right) u_{i}\left(b_{1}, s_{i}, s_{j}\right)$, which can only be positive if $u_{i}\left(b_{1}, s_{i}, s_{j}\right)$ is positive. Since $u_{i}$ is non-decreasing in $s_{j}$ by assumption, then $\delta_{i}\left(s_{i}, s_{j}\right)>0$ implies that $\delta_{i}\left(s_{i}, s_{j}^{\prime}\right) \geq 0$ for $s_{j}^{\prime}>s_{j}$. We must conclude that $\delta_{i}$ is weakly QSM in $s_{j}$. In fact, a somewhat more general result is supplied by the following lemma, which summarizes the preceding discussion.

Lemma 5 The following properties are true for almost every $s_{j}$ in $S_{j}$ :

(i) $\delta_{i}$ is non-decreasing in $s_{i}$. Moreover, $Q_{i}\left(b_{1}, s_{j}\right)>Q_{i}\left(b_{0}, s_{j}\right)$ implies that $\delta_{i}\left(s_{i}, s_{j}\right)<\delta_{i}\left(s_{i}^{\prime}, s_{j}\right)$ for $s_{i}<s_{i}^{\prime}$.

(ii) Suppose that $\beta_{j}$ is monotone at $b_{0}$. Then $\delta_{i}$ is weakly QSM in $s_{j}$.

The consequence of Lemma 5(i) is straightforward: $h_{i}$ is non-decreasing in its first argument, and it is strictly increasing whenever bidder $i$ 's probability of obtaining the object is strictly larger when he bids $b_{1}$ than when he bids $b_{0}$. In fact, it can be shown that $h_{i}$ is SSM in its first argument.

When the signals are independent $h_{i}$ is constant in its second argument. However, statistical dependence between signals establishes a probabilistic linkage between the signal observed by a bidder and the bids selected by the other bidder, which also affects the first bidder's expected payoff. In fact, the analysis of the dependence of $h_{i}$ on its second argument constitutes a more subtle problem whose structure depends crucially on the assumption that the signals are affiliated. To 
study this effect I rely on the following property of $M T P_{2}$ transformations (related results appear in Karlin [[4]]).

Lemma 6 Consider two mappings $\varphi: S_{j} \rightarrow \Re$ and $\psi: S_{i} \rightarrow \Re$ such that $\psi(x)=E\left(\varphi\left(\mathbf{s}_{j}\right) \mid \mathbf{s}_{i}=x\right)$. Suppose that the joint distribution of $\mathbf{s}_{i}$ and $\mathbf{s}_{j}$ satisfies assumption $A_{1}$ and that $\varphi$ is weakly QSM. Then $\psi$ is a QSM mapping.

We conclude directly from Lemma 5(ii) and Lemma 6 that $h_{i}$ is QSM in its second argument. Combining this with the fact that $h_{i}$ is $\mathrm{SSM}$ in its first argument, we establish the following.

Lemma 7 Let $b_{1}$ denote a winning bid for bidder $i$ and suppose that $\beta_{j}$ is monotone at $b_{0}$. Then $\pi_{i}\left(b_{1}, s_{i}\right)-\pi_{i}\left(b_{0}, s_{i}\right)$ is $S S M$ in $s_{i}$.

Proof. Define $h_{i}(x, y)=E\left(\delta_{i}\left(x, \mathbf{s}_{j}\right) \mid \mathbf{s}_{i}=y\right)$ and note that $h_{i}(x, x)=\pi_{i}\left(b_{1}, x\right)-$ $\pi_{i}\left(b_{0}^{+}, x\right)$. Suppose that $h_{i}(x, x) \geq 0$. Since $\beta_{j}$ is monotone at $b_{0}$, Lemma $5($ ii) implies that $\delta_{i}$ is weakly QSM in $s_{j}$. Then Lemma 6 implies $h_{i}$ is QSM in its second argument. We conclude that $h_{i}\left(x, x^{\prime}\right) \geq 0$ for $x^{\prime}>x$.

I claim that $Q_{i}\left(b_{1}, \mathbf{s}_{j}\right)-Q_{i}\left(b_{0}^{+}, \mathbf{s}_{j}\right)>0$ with positive probability with respect to the distribution of $\mathbf{s}_{j}$ conditional on $\mathbf{s}_{i}=x^{\prime}$. Arguing by contradiction, suppose $Q_{i}\left(b_{1}, \mathbf{s}_{j}\right)-Q_{i}\left(b_{0}^{+}, \mathbf{s}_{j}\right)=0$ with probability 1 . This means that $\delta_{i}\left(x, \mathbf{s}_{j}\right)=\left[u_{i}\left(b_{1}, x, \mathbf{s}_{j}\right)-u_{i}\left(b_{0}, x, \mathbf{s}_{j}\right)\right] Q_{i}\left(b_{1}, \mathbf{s}_{j}\right) \leq 0$ with probability 1 . But since $Q_{i}\left(b_{1}, \mathbf{s}_{j}\right)>0$ with positive probability because $b_{1}$ is a winning bid for $i$, we conclude that $\delta_{i}\left(x, \mathbf{s}_{j}\right)<0$ with positive probability. Thus, $h_{i}\left(x, x^{\prime}\right)<0$, a contradiction that establishes the claim.

Lemma $5(\mathrm{i})$ and the preceding claim imply that $\delta_{i}\left(x, \mathbf{s}_{j}\right) \leq \delta_{i}\left(x^{\prime}, \mathbf{s}_{j}\right)$ with probability 1 and that the inequality is strict with positive probability. Thus, $h_{i}\left(x^{\prime}, x^{\prime}\right)-h_{i}\left(x, x^{\prime}\right)=E\left(\delta_{i}\left(x^{\prime}, \mathbf{s}_{j}\right)-\delta_{i}\left(x, \mathbf{s}_{j}\right) \mid \mathbf{s}_{i}=x\right)>0$. We conclude that $h_{i}\left(x^{\prime}, x^{\prime}\right)>0$, as desired.

The following result is a corollary of Lemma 7.

Lemma 8 Suppose that $b$ is a winning bid for bidder $i$. Then $\pi_{i}\left(b, s_{i}\right)$ is $S S M$ in $s_{i}$.

Proof: Just select $b_{0}<\underline{b}$ and apply Lemma 7 .

\section{Monotonicity of the Equilibrium Strategies}

In this section I show that the equilibrium strategies are monotone increasing within the support of winning bids as long as the equilibrium satisfies the following regularity condition. 
Definition 1 Consider a mapping $\varphi: Y \rightarrow \Re$, where $Y \subset \Re$. For each $\lambda \in \Re$, define the mapping $\varphi_{\lambda}: Y \rightarrow \Re$ such that $\varphi_{\lambda}(y)=\varphi(y)-\lambda$. I say that $\varphi$ is piecewise monotone if, for each $\lambda \in \Re$ and each compact set $K \subset Y$, the restriction of $\varphi_{\lambda}$ to $K$ changes sign finitely many times.

For example, if $\varphi:[a, b] \rightarrow \Re$ is a differentiable function, it is piecewise monotone if and only if the sign of the derivative changes finitely many times. On the other hand, the differentiable function $\varphi(y)=y^{2} \sin (1 / y)$ defined on $[-1,1]$ is not piecewise monotone. Finally note that when $Y$ is a discrete set, $\varphi$ is always piecewise monotone.

Definition 2 I say that the strategy $\beta_{i}$ is regular if almost every selection $\tilde{\beta}_{i}$ from $\beta_{i}$ is a piecewise monotone function. A regular equilibrium is a Nash equilibrium in which both bidders employ regular strategies.

The following lemma shows that if a bidder's strategy is monotone at each point of the interval $\left(\underline{b}, b_{0}\right)$, a regular best response strategy by the other bidder must be monotone at each point of a larger interval $\left(\underline{b}, b_{0}+\varepsilon\right)$. The argument heavily relies on the affiliation related properties derived in the preceding section. The following illustration contains the essence of the argument. Suppose that both bidders' strategies are monotone at each bid lower than $b_{0}$, where $b_{0}>\underline{b}$. Also suppose that there are two signals $x_{0}$ and $x_{1}$, with $x_{0}>x_{1}$, and a bid $b_{1}>b_{0}$ such that $b_{0}$ is optimal for bidder $i$ when his signal is $x_{0}$ and $b_{1}$ is optimal when his signal is $x_{1}$. Thus, in this scenario bidder $i$ 's strategy is not monotone at $b_{0}$. However, since $b_{1}$ is optimal for $i$ when his signal is $x_{1}$, we must have that $\pi_{i}\left(b_{1}, x_{1}\right) \geq \pi_{i}\left(b_{0}, x_{1}\right)$, and then Lemma 7 implies that $\pi_{i}\left(b_{1}, x_{0}\right)>\pi_{i}\left(b_{0}, x_{0}\right)$, contradicting the optimality of $b_{0}$ when $i$ 's signal is $x_{0}$.

A detailed proof of the following result is included in the appendix.

Lemma 9 Let $\beta$ denote a regular equilibrium. Suppose that $\beta_{j}$ is monotone at $b_{0}$. Then there is some $\varepsilon>0$ such that both $\beta_{i}$ and $\beta_{j}$ are monotone at each bid $b^{\prime} \in\left(b_{0}, b_{0}+\varepsilon\right)$. Moreover, if $b_{0}>\underline{b}, \beta_{i}$ also is monotone at $b_{0}$.

Proof. We argue by contradiction. Suppose that for every $\varepsilon>0$, there is some $b \in\left(b_{0}, b_{0}+\varepsilon\right)$ such that $\beta_{i}$ is not monotone at $b$. Since $\beta_{i}$ is a regular strategy, this can only happen if there is a sequence $\left(b^{n}, x^{n}\right)_{n=1, \ldots, \infty}$ such that $x^{n} \in \beta_{i}^{-1}\left(b^{n}\right)$ and $\lim _{n \rightarrow \infty}\left(b^{n}, x^{n}\right)=\left(b_{0}, x_{0}\right)$, where $x_{0}>x_{1} \in \beta_{i}^{-1}\left(b_{1}\right)$ and $b_{0}<b_{1}$.

Note that $x_{1} \in \beta_{i}^{-1}\left(b_{1}\right)$ implies that $\pi_{i}\left(b_{1}, x_{1}\right) \geq \pi_{i}\left(b_{0}^{+}, x_{1}\right)$. Since $\beta_{j}$ is monotone at $b_{0}$ and $x_{0}>x_{1}$, Lemma 7 implies that $\pi_{i}\left(b_{1}, x_{0}\right)>\pi_{i}\left(b_{0}^{+}, x_{0}\right)$. Thus, $\pi_{i}\left(b_{1}, x^{n}\right)>\pi_{i}\left(b^{n}, x^{n}\right)$ for $n$ sufficiently large. We conclude that $x^{n} \notin \beta_{i}^{-1}\left(b^{n}\right)$. This contradiction proves that $\beta_{i}$ is monotone at each bid $b^{\prime} \in\left(b_{0}, b_{0}+\varepsilon\right)$, for some $\varepsilon>0$. An identical argument implies that also $\beta_{j}$ is monotone at each bid $b^{\prime} \in\left(b_{0}, b_{0}+\varepsilon\right)$. To see that $\beta_{i}$ also is monotone at $b_{0}$ when $b_{0}>\underline{b}$, just notice 
that this strategy is atomless at $b_{0}$ in this case.

Remark. Suppose that both bidders' strategies are monotone at $b_{0}$ but, for every $\varepsilon>0$, there is some $b \in\left(b_{0}, b_{0}+\varepsilon\right)$ such that $\beta_{i}$ is not monotone at $b$. If $\beta_{i}$ is not a regular strategy, still there may not exist any pair $\left(b_{1}, x_{1}\right)$ and any sequence $\left(b^{n}, x^{n}\right)_{n=1, . ., \infty}$, both in the graph of $\operatorname{supp} \beta_{i}$, such that $\lim _{n \rightarrow \infty} b^{n}=b_{0}<b_{1}$ and $\lim _{n \rightarrow \infty} x^{n}>x_{1} \in \beta_{i}^{-1}\left(b_{1}\right)$. An example is the case of a strategy $\beta_{i}$ that is monotone at $b_{0}$ with $\beta_{i}^{-1}\left(b_{0}\right)=\left\{x_{0}\right\}$ and oscillates infinitely many times between the bounds $b_{0}+\gamma\left(s_{i}-x_{0}\right)$ and $b_{0}+2 \gamma\left(s_{i}-x_{0}\right)$ in the interval $\left(x_{0}, x_{0}+\varepsilon\right)$ in $S_{i}$, for every $\varepsilon>0$. Clearly, in this case the argument employed in the proof of Lemma 9 does not go through.

A variation of the preceding argument allows us to restrict equilibrium behavior close to the infimum of the support of winning bids.

Lemma 10 Either $\beta_{1}$ or $\beta_{2}$ is monotone at $\underline{b}$.

Combining Lemma 9 and Lemma 10, we reach the conclusion that regular equilibrium strategies are monotone increasing over the whole interior of the support of winning bids. Our main result follows.

Proposition 1 Let $\beta$ denote a regular equilibrium and also denote $S_{i}^{0}=\left\{s_{i} \in\right.$ $\left.S_{i}: s_{i}>\sup \beta_{i}^{-1}(\underline{b})\right\}$. Then, for $i=1,2$, we have that $\beta_{i}\left(s_{i}\right) \leq \underline{b}$ with probability 1 when $s_{i} \notin S_{i}^{0}$, and also that the restriction of $\beta_{i}$ to $S_{i}^{0}$ is monotone increasing. In other words, at every regular equilibrium, the strategy employed by each bidder is monotone at each winning bid.

Proof. Denote $m=\inf \left\{b>\underline{b}:\right.$ either $\beta_{1}$ or $\beta_{2}$ is not monotone at $\left.b\right\}$. First, I rule out the possibility that $m=\underline{b}$. In fact, Lemma 10 shows that the strategy of at least one of the bidders, say $j$, is monotone at $\underline{b}$. Then Lemma 9 implies that both bidders' strategies are monotone at each bid $b \in(\underline{b}, \underline{b}+\varepsilon)$, for some $\varepsilon>0$. This implies that $m>\underline{b}$. Now suppose that $\underline{b}<m \leq \bar{b}$. By definition of $m$ both $\beta_{1}$ and $\beta_{2}$ are monotone at each $b \in(\underline{b}, m)$. Since $m>\underline{b}$, Lemma 4 implies that both $\beta_{1}$ and $\beta_{2}$ are monotone at $m$. Again, Lemma 9 implies that both $\beta_{1}$ and $\beta_{2}$ are monotone at each $b \in(\underline{b}, m+\varepsilon)$, for some $\varepsilon>0$. Since this contradicts the definition of $m$, we must conclude that $m=\infty$, as desired.

Since every strategy is regular when the signals are discrete valued, the following corollary follows.

Corollary 1 Suppose that $S_{1}$ and $S_{2}$ are finite sets. At any equilibrium $\beta$, $\beta_{i}\left(s_{i}\right) \leq \underline{b}$ with probability 1 when $s_{i} \notin S_{i}^{0}$ and the restriction of $\beta_{i}$ to $S_{i}^{0} i s$ monotone increasing, for $i=1,2$. 


\section{Uniqueness of the Equilibrium}

In this section I investigate the uniqueness of the equilibrium in the symmetric case. Maskin and Riley [[7]] establish uniqueness when the bidders use monotone strategies and the set of signals is a closed interval. Their analysis requires the following additional assumptions.

$\left(A_{4}\right) \quad$ (i) $S_{1}=S_{2}=S$, (ii) $u_{i}\left(b, s_{i}, s_{j}\right)=u_{j}\left(b, s_{i}, s_{j}\right)=u\left(b, s_{i}, s_{j}\right)$, (iii) $F$ is a symmetric distribution, and (iv) $s_{i}>s_{j} \Rightarrow u\left(b, s_{i}, s_{j}\right) \geq u\left(b, s_{j}, s_{i}\right)$.

$\left(A_{5}\right) \quad(\mathrm{i}) \frac{\partial}{\partial b} u\left(b, s_{i}, s_{j}\right)$ is a non-increasing function, and (ii) $\frac{\partial^{2}}{\partial b \partial s_{i}} u\left(b, s_{i}, s_{j}\right) \leq$ $\frac{\partial^{2}}{\partial b \partial s_{j}} u\left(b, s_{i}, s_{j}\right)$.

$\left(A_{6}\right) \quad f\left(s_{i} \mid s_{j}\right) / F\left(s_{i} \mid s_{j}\right)$ is strictly decreasing in $s_{i}$.

The following lemma shows that symmetry implies additional restrictions at the upper lower bound of the support of winning bids.

Lemma 11 Suppose that $r=0$ and that assumption $A_{4}$ is satisfied. Then, at every regular equilibrium, $\underline{b}_{1}=\underline{b}_{2}=\underline{b}$. Moreover, (i) when $S=[\underline{s}, \bar{s}]$, no bidder has a mass point at $\underline{b}$, and (ii) when $S=\{\underline{s}, . ., \bar{s}\}$, we have that $\beta_{i}^{-1}(\underline{b})=$ $\beta_{j}^{-1}(\underline{b})=\{\underline{s}\}$ and that the type $\underline{s}$ of at least one player bids $\underline{b}$ with probability 1 , where $u(\underline{b}, \underline{s}, \underline{s})=0$.

Maskin and Riley's argument relies on the idea that if the type of buyer $i$ that bids $b$ is larger than the type of buyer $j$ that bids $b$, then the type of buyer $i$ must be larger for every bid higher than $b$. This contradicts the requirement that $\beta_{i}^{-1}(\bar{b})=\beta_{j}^{-1}(\bar{b})=\{\bar{s}\}$ and shows that the unique equilibrium in monotone strategies must be the symmetric one. I also provide an extension of this result to the case of discrete-valued signals. Combining these results with Proposition 1 , I obtain the following.

Proposition 2 (i) Suppose that assumptions $A_{1}-A_{6}$ are satisfied and that $S=$ $[\underline{s}, \bar{s}]$. Then there is a unique regular equilibrium. (ii) Suppose that assumptions $A_{1}-A_{4}$ are satisfied and that $S=\{\underline{s}, \underline{s}+1, . ., \bar{s}\}$. Then the equilibrium is unique.

\section{Proof.}

(i) It follows directly from Proposition 1 and Theorem 3 in Maskin and Riley [[7]].

(ii) Since every strategy is regular when $S$ is finite, Corollary 1 and Lemma 11 imply that the equilibrium strategies are monotone over the set $S$. Now, I claim that the equilibrium is symmetric. I argue by contradiction. Suppose that there is a bid $b_{*}$ and a type $s_{*}$ such that $Q_{j}\left(b_{*}, s_{*}\right)>Q_{i}\left(b_{*}, s_{*}\right)$. Since in equilibrium $Q_{j}(\bar{b}, \bar{s})=Q_{i}(\bar{b}, \bar{s})=1$, by Lemma 3 , and the strategies are monotone, there 
must exist some bids $b_{0}$ and $b_{1}$, with $b_{0}<b_{1}$, and some type $x \in S$ such that $\beta_{i}^{-1}(b)=\beta_{j}^{-1}(b)=\{x\}$ for every $b \in\left[b_{0}, b_{1}\right]$ and

$$
\begin{aligned}
& Q_{j}\left(b_{0}, x\right)>Q_{i}\left(b_{0}, x\right) \\
& Q_{j}\left(b_{1}, x\right) \leq Q_{i}\left(b_{1}, x\right)
\end{aligned}
$$

Note that $\pi_{i}(b, x)=u(b, x, x) Q_{i}(b, x) f(x \mid x)+\sum_{s=\underline{s}}^{x-1} u(b, x, s) f(s \mid x)$, where $f$ is a probability mass function. Thus,

$$
\begin{aligned}
& {\left[\pi_{i}\left(b_{1}, x\right)-\pi_{i}\left(b_{0}, x\right)\right]-\left[\pi_{j}\left(b_{1}, x\right)-\pi_{j}\left(b_{0}, x\right)\right]=} \\
& =\left\{u\left(b_{1}, x, x\right)\left[Q_{i}\left(b_{1}, x\right)-Q_{j}\left(b_{1}, x\right)\right]-u\left(b_{0}, x, x\right)\left[Q_{i}\left(b_{0}, x\right)-Q_{j}\left(b_{0}, x\right)\right\} f(x \mid x)\right.
\end{aligned}
$$

Note that $\pi_{i}\left(b_{1}, x\right) \geq 0$ implies that $u\left(b_{1}, x, x\right) \geq 0$ and that $u\left(b_{0}, x, x\right)>0$. Thus, (2) implies that the expression (3) is strictly positive. Then either $\pi_{i}\left(b_{1}, x\right)-$ $\pi_{i}\left(b_{0}, x\right)>0$ or $\pi_{j}\left(b_{1}, x\right)-\pi_{j}\left(b_{0}, x\right)>0$, contradicting the definition of $x$. This establishes the claim.

Finally, Lemma 11(ii) shows that at a symmetric equilibrium both players bid $\underline{b}$ with probability 1 when their type is $\underline{s}$, where $\underline{b}$ is determined by the condition $u(\underline{b}, \underline{s}, \underline{s})=0$. Thus, monotonicity and the requirement that $Q_{j}(\bar{b}, \bar{s})=Q_{i}(\bar{b}, \bar{s})$ uniquely determine the equilibrium strategies.

\section{Final Remarks}

The monotonicity result derived in this paper provides the missing link for the analysis of uniqueness in two-person first price auctions. At least in the twoperson case the assumption of affiliation provides sufficient structure to ensure a very general monotonicity result, which is independent of any symmetry considerations and of the bidders' attitudes toward risk. Combining this result with earlier work by Maskin and Riley, I also establish a uniqueness result for the two-person symmetric case.

The two main limitations of the results reported here are related to our focus on regular equilibria of two-person auctions. The restriction to regular equilibria is of a technical nature but may not be such a demanding requirement from the economic point of view. Moreover, it is not restrictive at all when the signals are discrete valued. The second qualification is of a more fundamental nature and, in fact, our results can be considered only a preliminary step in the analysis of the $n$-person case. However, the extension of the methods used here to the general case does not seem to be straightforward. In particular, Lemma 6 does not generalize to the $n$-dimensional case. The following counterexample establishes this point. Consider a probability measure $p$ defined on the set $\Theta$ of the 3 tuples $(i, j, k)$ such that $i, j, k \in\{0,1\}$. Let $p_{i j k}$ denote the probability of the element $(i, j, k)$ and let $p_{i j}(k)$ denote the conditional probability that the first two elements take values $i$ and $j$ given the fact that the third one takes the value 
$k$. Suppose that $p_{00}(0)=p_{01}(0)=1 / 3, p_{10}(0)=p_{11}(0)=1 / 6, p_{00}(1)=4 / 15$, $p_{01}(1)=4 / 10, p_{10}(1)=2 / 15$ and $p_{11}(1)=2 / 10$. Define a function $\varphi$ on $\Theta$ such that $\varphi(i, j, k)=\varphi_{i j}$. It can be easily verified that $p$ is $M T P_{2}$ and that $\varphi$ is a weakly $Q S M$ mapping. Finally, notice that $E(\varphi \mid k=0)=0$ and that $E(\varphi \mid k=1)=-0.06$. Thus, $E(\varphi \mid k)$ is not $Q S M$.

\section{Appendix}

Proof of Lemma 2. Using the continuity of $u_{i}$, we can write

$$
\begin{aligned}
& \pi_{i}(\hat{b}, x)-\pi_{i}\left(\hat{b}^{-}, x\right)=E\left(u_{i}\left(\hat{b}, \mathbf{s}_{i}, \mathbf{s}_{j}\right)\left[Q_{i}\left(\hat{b}, \mathbf{s}_{j}\right)-Q_{i}\left(\hat{b}^{-}, \mathbf{s}_{j}\right)\right] \mid \mathbf{s}_{i}=x\right) \\
& \pi_{i}\left(\hat{b}^{+}, x\right)-\pi_{i}(\hat{b}, x)=E\left(u_{i}\left(\hat{b}, \mathbf{s}_{i}, \mathbf{s}_{j}\right)\left[Q_{i}\left(\hat{b}^{+}, \mathbf{s}_{j}\right)-Q_{i}\left(\hat{b}, \mathbf{s}_{j}\right)\right] \mid \mathbf{s}_{i}=x\right)
\end{aligned}
$$

Note that $Q_{i}\left(\hat{b}, s_{j}\right)-Q_{i}\left(\hat{b}^{-}, s_{j}\right)=Q_{i}\left(\hat{b}^{+}, s_{j}\right)-Q_{i}\left(\hat{b}, s_{j}\right)$ because the tie-breaker is symmetric. Thus, $\pi_{i}(\hat{b}, x)-\pi_{i}\left(\hat{b}^{-}, x\right)=\pi_{i}\left(\hat{b}^{+}, x\right)-\pi_{i}(\hat{b}, x)$. On the other hand, $\pi_{i}(\hat{b}, x)-\pi_{i}\left(\hat{b}^{-}, x\right) \geq 0$ and $\pi_{i}\left(\hat{b}^{+}, x\right)-\pi_{i}(\hat{b}, x) \leq 0$ because of the optimality of $\hat{b}$ for the bidder $i$ with signal $x$. We conclude that $\pi_{i}(\hat{b}, x)=\pi_{i}\left(\hat{b}^{-}, x\right)=\pi_{i}\left(\hat{b}^{+}, x\right)$, as desired.

Proof of Lemma 3. I argue by contradiction. Suppose that the bid $\hat{b} \in(\underline{b}, \bar{b}]$ is optimal for bidder $i$ with signal $x$ and that bidder $j$ bids with probability zero in some interval $[\hat{b}-\varepsilon, \hat{b})$. Since $\pi_{i}(b, x)$ is continuous in $\hat{b}$ by Lemma 2 , an arbitrarily small reduction in bidder $i$ 's bid has an arbitrarily small effect on his expected payoff. However, an additional reduction in his bid to $\hat{b}-\varepsilon$ means a definite reduction in what he expects to pay if he wins and does not affect his chance of winning. Thus, $\pi_{i}(\hat{b}, x)<\pi_{i}(\hat{b}-\varepsilon, x)$, contradicting the optimality of $\hat{b}$.

Proof of Lemma 4. Suppose that $\beta_{j}$ has a mass point and is quasi-monotone at $b$. Consider a sequence $\left(b^{n}, x^{n}\right)_{n \rightarrow \infty}$ such that $b^{n} \uparrow b$ and $x^{n} \in \beta_{i}^{-1}\left(b^{n}\right)$ (existence of such sequence follows from Lemma 3$)$. Denote $x=\lim _{n \rightarrow \infty} x^{n}$ and $\tilde{s}_{j}=\sup \left\{s_{j} \in S_{j}: Q_{i}\left(b^{-}, s_{j}\right)>0\right\}$. The fact that $\beta_{j}$ is quasi-monotone at $b$ implies that $Q_{i}\left(b^{-}, s_{j}\right)=0$ for $s_{j}>\tilde{s}_{j}$. Thus, $\lim _{n \rightarrow \infty} \pi_{j}\left(b^{n}, x^{n}\right)=\pi\left(b^{-}, x\right)=$ $E\left(u_{i}\left(b, \mathbf{s}_{i}, \mathbf{s}_{j}\right) Q_{i}\left(b^{-}, \mathbf{s}_{j}\right) \mid \mathbf{s}_{i}=x, \mathbf{s}_{j} \leq \tilde{s}_{j}\right) P\left(\mathbf{s}_{j} \leq \tilde{s}_{j} \mid \mathbf{s}_{i}=x\right)$. Note that $\pi\left(b^{-}, x\right) \geq$ 0 as a consequence of the fact that $\pi_{j}\left(b^{n}, x^{n}\right) \geq 0$ for every $n$. Also note that $P\left(\mathbf{s}_{j} \leq \tilde{s}_{j} \mid \mathbf{s}_{i}=x\right)>0$ due to the fact that $b>\underline{b}$. Hence, we conclude that $u_{i}\left(b, x, \tilde{s}_{j}\right) \geq 0$.

If $u_{i}\left(b, x, \tilde{s}_{j}\right)=0$, then $\pi_{i}\left(b^{-}, x\right)=0$ and $\pi_{i}(b-\varepsilon, x)>0$ for some small $\varepsilon>0$. Thus, for $n$ sufficiently large, $\pi_{i}\left(b-\varepsilon, x^{n}\right)>\pi_{i}\left(b^{n}, x^{n}\right) \cong 0$, which implies that $x^{n} \notin \beta_{i}^{-1}\left(b^{n}\right)$, a contradiction.

If $u_{i}\left(b, x, \tilde{s}_{j}\right)>0$, then $u_{i}\left(b, x, s_{j}\right)>0$ for every $s_{j}>\tilde{s}_{j}$. Thus,

$$
E\left(u_{i}\left(b, \mathbf{s}_{i}, \mathbf{s}_{j}\right) Q_{i}\left(b, \mathbf{s}_{j}\right) \mid \mathbf{s}_{i}=x, \mathbf{s}_{j}>\tilde{s}_{j}\right)>0
$$


Note that $Q_{i}\left(b^{-}, s_{j}\right)=0$ for $s_{j}>\tilde{s}_{j}$ and $Q_{i}\left(b, s_{j}\right)=Q_{i}\left(b^{-}, s_{j}\right)$ for $s_{j}<\tilde{s}_{j}$ as a consequence of the fact that $\beta_{j}$ is quasi-monotone at $b$. Using also the continuity of $u_{i}$ in $b$ we can write

$$
\begin{aligned}
& \pi_{i}(b, x)-\pi_{i}\left(b^{-}, x\right)=E\left(u_{i}\left(b, \mathbf{s}_{i}, \mathbf{s}_{j}\right)\left[Q_{i}\left(b, \mathbf{s}_{j}\right)-Q_{i}\left(b^{-}, \mathbf{s}_{j}\right)\right] \mid \mathbf{s}_{i}=x\right) \\
& \quad=E\left(u_{i}\left(b, \mathbf{s}_{i}, \mathbf{s}_{j}\right)\left[Q_{i}\left(b, \mathbf{s}_{j}\right)-Q_{i}\left(b^{-}, \mathbf{s}_{j}\right)\right] \mid \mathbf{s}_{i}=x, \mathbf{s}_{j}>\tilde{s}_{j}\right) P\left(\mathbf{s}_{j}>\tilde{s}_{j} \mid \mathbf{s}_{i}=x\right) \\
& \quad=E\left(u_{i}\left(b, \mathbf{s}_{i}, \mathbf{s}_{j}\right) Q_{i}\left(b, \mathbf{s}_{j}\right) \mid \mathbf{s}_{i}=x, \mathbf{s}_{j}>\tilde{s}_{j}\right) P\left(\mathbf{s}_{j}>\tilde{s}_{j} \mid \mathbf{s}_{i}=x\right)>0
\end{aligned}
$$

where the last inequality follows from (4) and the fact that $P\left(\mathbf{s}_{j}>\tilde{s}_{j} \mid \mathbf{s}_{i}=x\right)>$ 0 because of the mass point at $b$. Since $\pi_{i}\left(b, x^{n}\right)-\pi_{i}\left(b^{n}, x^{n}\right) \rightarrow \pi_{i}(b, x)-$ $\pi_{i}\left(b^{-}, x\right)>0$ as $n \rightarrow \infty$, we conclude that $x^{n} \notin \beta_{i}^{-1}\left(b^{n}\right)$ also in this case. This contradiction establishes the lemma.

\section{Proof of Lemma 5.}

(i) Follows directly from equation (1).

(ii) First, I claim that $\delta_{i}(s)>0$ implies that (a) $Q_{i}\left(b_{0}^{+}, s_{j}\right)<1$ and (b) $u_{i}\left(b_{1}, s\right)>$ 0 . To see this, note that $Q_{i}\left(b_{0}^{+}, s_{j}\right)=1$ implies that $Q_{i}\left(b_{1}, s_{j}\right)=1$ and therefore that $\delta_{i}(s)=u_{i}\left(b_{1}, s\right)-u_{i}\left(b_{0}, s\right)<0$, so (a) follows. Now suppose that $u_{i}(b, s) \leq 0$. Since $\left|u_{i}\left(b_{1}, s\right)\right|>\left|u_{i}\left(b_{0}, s\right)\right|$ when $u_{i}\left(b_{0}, s\right) \leq 0$, we have that $\delta_{i}(s)=-\left|u_{i}\left(b_{1}, s\right)\right| Q_{i}\left(b_{1}, s_{j}\right)-u_{i}\left(b_{0}, s\right) Q_{i}\left(b_{0}^{+}, s_{j}\right) \leq 0$. This establishes the claim.

Now suppose that $\delta_{i}(s)>0$ and consider a signal $s_{j}^{\prime}>s_{j}$. Since $\beta_{j}$ is monotone at $b_{0}$, Lemma 1 and the preceding claim imply that $Q_{i}\left(b_{0}^{+}, s_{j}^{\prime}\right)=0$. Thus, we have that $\delta_{i}\left(s_{i}, s_{j}^{\prime}\right)=Q_{i}\left(b_{1}, s_{j}^{\prime}\right) u_{i}\left(b_{1}, s_{i}, s_{j}^{\prime}\right) \geq 0$, where the inequality follows from the preceding claim and the fact that $u_{i}\left(b_{1}, s_{i}, s_{j}^{\prime}\right) \geq u_{i}\left(b_{1}, s_{i}, s_{j}\right)$ by assumption $A_{2}$. We conclude that $\delta_{i}\left(s_{i}, s_{j}\right)>0$ implies that $\delta_{i}\left(s_{i}, s_{j}^{\prime}\right) \geq 0$, as desired.

Proof of Lemma 6. Since $\varphi$ is weakly QSM, there must be a parameter value $s_{j}^{0} \in S_{j}$ such that $\varphi\left(s_{j}\right)\left(s_{j}-s_{j}^{0}\right) \geq 0$ for almost every $s_{j} \in S_{j}$. Given $x, x^{\prime} \in S_{i}$ such that $x<x^{\prime}$, denote $\Delta(s)=f_{j}\left(s_{j} \mid x^{\prime}\right) / f_{j}\left(s_{j}^{0} \mid x^{\prime}\right)-f_{j}\left(s_{j} \mid x\right) / f_{j}\left(s_{j}^{0} \mid x\right)$, where $f_{j}$ denotes the density of the conditional distribution of $\mathbf{s}_{j}$ with respect to $\mathbf{s}_{i}$. I claim that $\varphi\left(s_{j}\right) \Delta\left(s_{j}\right) \geq 0$ for almost every $s_{j} \in S_{j}$. Note that when $s_{j}<s_{j}^{0}$ we have that $\Delta\left(s_{j}\right) \leq 0$ because $f$ is $M T P_{2}$ and that $\varphi\left(s_{j}\right) \leq 0$ because $\varphi$ is weakly QSM. Similarly, $\Delta\left(s_{j}\right) \geq 0$ and $\varphi\left(s_{j}\right) \geq 0$ when $s_{j}>s_{j}^{0}$. The claim follows. Finally, note that $\psi\left(x^{\prime}\right) / f_{j}\left(s_{j}^{0} \mid x^{\prime}\right)-\psi(x) / f_{j}\left(s_{j}^{0} \mid x\right)=\int_{S_{j}} \varphi(\eta) \Delta(\eta) d \eta \geq 0$. Thus, $\psi$ is QSM.

Proof of Lemma 10. For concreteness, suppose that $\underline{b}_{j} \geq \underline{b}_{i}$. First, I claim that $\beta_{j}$ is quasi-monotone at $\underline{b}$. This is trivially true when $\underline{b}_{j}=\underline{b}$. On the other hand, when $\underline{b}_{j}<\underline{b}, \underline{b}$ is a winning bid for bidder $j$. If this bid is optimal for $j$ when his signal is $s_{j}$, we must have that $\pi_{j}\left(\underline{b}, s_{j}\right) \geq 0$. Thus, Lemma 8 implies that $\pi_{j}\left(\underline{b}, s_{j}^{\prime}\right)>0$ for $s_{j}^{\prime}>s_{j}$. Since bids lower than $\underline{b}$ yield zero expected profits, we conclude that when bidder $j$ 's signal is larger than $s_{j}$, he bids at least $\underline{b}$. The claim follows. 
Since $\beta_{j}$ is quasi-monotone at $\underline{b}$, the lemma follows trivially if $\beta_{j}$ is atomless at $\underline{b}$. Consider the case in which $\beta_{j}$ has a mass point at $\underline{b}$. Since in this case $\underline{b}$ is a winning bid for $i$ and $\beta_{j}$ is quasi-monotone at $\underline{b}$, Lemma 8 and the argument employed in the preceding paragraph imply that $\beta_{i}$ also is quasi-monotone at $\underline{b}$. To show that $\beta_{i}$ is actually monotone at $\underline{b}$, we only need to show that $\beta_{i}^{-1}(\underline{b})$ is a singleton. We argue by contradiction. Suppose that $\beta_{i}^{-1}(\underline{b})$ contains two signals $s_{i}$ and $s_{i}^{\prime}$ such that $s_{i}^{\prime}>s_{i}$. Since $\pi_{i}\left(\underline{b}, s_{i}\right) \geq 0$, Lemma 8 implies that $\pi_{i}\left(\underline{b}, s_{i}^{\prime}\right)>0$. Moreover, since $\beta_{j}$ is quasi-monotone at $\underline{b}$, the types of bidder $j$ that bid $\underline{b}$ are larger than the ones that bid less than $\underline{b}$. Using these observations and the fact that $u_{i}$ is non-decreasing in $s_{j}$, an argument similar to the one used in the proof of Lemma 4 implies that $E\left(u_{i}\left(b, \mathbf{s}_{i}, \mathbf{s}_{j}\right) Q_{i}\left(b, \mathbf{s}_{j}\right) \mid \mathbf{s}_{i}=s_{j}^{\prime}, \mathbf{s}_{j}>\tilde{s}_{j}\right)>0$, where $\tilde{s}_{j}=\sup \left\{s_{j} \in S_{j}: Q_{i}\left(b^{-}, s_{j}\right)>0\right\}$. Thus,

$$
\begin{aligned}
& \pi_{i}\left(b^{+}, s_{j}^{\prime}\right)-\pi_{i}\left(b, s_{j}^{\prime}\right)=E\left(u_{i}\left(b, \mathbf{s}_{i}, \mathbf{s}_{j}\right)\left[Q_{i}\left(b^{+}, \mathbf{s}_{j}\right)-Q_{i}\left(b, \mathbf{s}_{j}\right)\right] \mid \mathbf{s}_{i}=s_{j}^{\prime}\right) \\
& \quad=E\left(u_{i}\left(b, \mathbf{s}_{i}, \mathbf{s}_{j}\right)\left[Q_{i}\left(b^{+}, \mathbf{s}_{j}\right)-Q_{i}\left(b, \mathbf{s}_{j}\right)\right] \mid \mathbf{s}_{i}=s_{j}^{\prime}, \mathbf{s}_{j}>\tilde{s}_{j}\right) P\left(\mathbf{s}_{j}>\tilde{s}_{j} \mid \mathbf{s}_{i}=s_{j}^{\prime}\right) \\
& \quad=E\left(u_{i}\left(b, \mathbf{s}_{i}, \mathbf{s}_{j}\right)\left[Q_{i}\left(b, \mathbf{s}_{j}\right)-Q_{i}\left(b^{-}, \mathbf{s}_{j}\right)\right] \mid \mathbf{s}_{i}=s_{j}^{\prime}, \mathbf{s}_{j}>\tilde{s}_{j}\right) P\left(\mathbf{s}_{j}>\tilde{s}_{j} \mid \mathbf{s}_{i}=s_{j}^{\prime}\right) \\
& \quad=E\left(u_{i}\left(b, \mathbf{s}_{i}, \mathbf{s}_{j}\right) Q_{i}\left(b, \mathbf{s}_{j}\right) \mid \mathbf{s}_{i}=s_{j}^{\prime}, \mathbf{s}_{j}>\tilde{s}_{j}\right) P\left(\mathbf{s}_{j}>\tilde{s}_{j} \mid \mathbf{s}_{i}=s_{j}^{\prime}\right)>0
\end{aligned}
$$

where we used the facts that $Q_{i}\left(\hat{b}, s_{j}\right)-Q_{i}\left(\hat{b}^{-}, s_{j}\right)=Q_{i}\left(\hat{b}^{+}, s_{j}\right)-Q_{i}\left(\hat{b}, s_{j}\right)$ because the tie-breaker is symmetric, and that $Q_{i}\left(b^{-}, s_{j}\right)=0$ for $s_{j}>\tilde{s}_{j}$ and $Q_{i}\left(b, s_{j}\right)=Q_{i}\left(b^{+}, s_{j}\right)$ for $s_{j}<\tilde{s}_{j}$ as a consequence of the fact that $\beta_{j}$ is quasimonotone at $b$. But (5) implies that $s_{j}^{\prime} \notin \beta_{i}^{-1}(\underline{b})$. This contradiction completes the proof.

Proof of Lemma 11. I argue by contradiction. Let $\underline{b}_{i}<\underline{b}_{j}=\underline{b}$ and denote $s_{i}^{0}=\sup \beta_{i}^{-1}(\underline{b})$. First I claim that $u\left(\underline{b}, s_{i}^{0}, \underline{s}\right) \leq 0$. To establish the claim suppose that $u\left(\underline{b}, s_{i}^{0}, \underline{s}\right)>0$. Then $\pi_{i}\left(\underline{b}+\varepsilon, s_{i}^{0}\right)>\pi_{i}\left(\underline{b}, s_{i}^{0}\right)$ for a sufficiently small $\varepsilon>0$, which contradicts the optimality of $\underline{b}$ for $i$ when his signal is $s_{i}^{0}$. The claim follows.

Take $\underline{b}_{i}<b<\underline{b}$. Then

$$
\begin{aligned}
& \pi_{j}(\underline{b}, \underline{s})-\pi_{j}(b, \underline{s})= \\
& =E\left(u\left(\underline{b}, \underline{s}, \mathbf{s}_{i}\right)\left[Q_{j}\left(\underline{b}, \mathbf{s}_{i}\right)-Q_{j}\left(b, \mathbf{s}_{i}\right)\right]+Q_{j}\left(b, \mathbf{s}_{i}\right)\left[u\left(\underline{b}, \underline{s}, \mathbf{s}_{i}\right)-u\left(b, \underline{s}, \mathbf{s}_{i}\right)\right] \mid \mathbf{s}_{i} \leq s_{i}^{0}\right)
\end{aligned}
$$

The preceding claim and assumption $A_{4}$ imply that $u\left(\underline{b}, \underline{s}, s_{i}^{0}\right) \leq 0$. By assumption $A_{3}$, we have that $u\left(\underline{b}, \underline{s}, \mathbf{s}_{i}\right)-u\left(b, \underline{s}, \mathbf{s}_{i}\right)<0$. Moreover, $Q_{j}\left(b, \mathbf{s}_{i}\right)>0$ with positive probability since $\underline{b}_{i}<b$. Thus, equation 6 implies that $\pi_{j}(\underline{b}, \underline{s})-\pi_{j}(b, \underline{s})<0$, so $\underline{s} \notin \beta_{j}^{-1}(\underline{b})$. This contradicts Proposition 1 and establishes the first assertion.

To establish the part (i) of the last assertion, suppose that $S=[\underline{s}, \bar{s}]$. Also suppose that $\beta_{j}$ has a mass point at $\underline{b}$. Since $\underline{b}$ is a winning bid for bidder $i$, we have that $\pi_{i}\left(\underline{b}^{+}, \underline{s}\right) \geq 0$. This implies that $u\left(\underline{b}, \underline{s}, \hat{s}_{j}\right)>0$ for some $\hat{s}_{j} \in \beta_{j}^{-1}(\underline{b})$. Thus, assumption $A_{4}$ implies that $u\left(\underline{b}, \hat{s}_{j}, \underline{s}\right)>0$. But then $\pi_{j}\left(\underline{b}+\varepsilon, \hat{s}_{j}\right)>\pi_{j}\left(\underline{b}, \hat{s}_{j}\right)$ for a sufficiently small $\varepsilon>0$, a contradiction that establishes (i). 
Finally, consider part (ii). Suppose that $S=\{\underline{s}, . ., \bar{s}\}$. First I claim that both bidders' strategies have a mass point at $\underline{b}$. To show this, I argue by contradiction. Suppose $\beta_{j}$ is atomless at $\underline{b}$. Since the strategies are monotone, there must exist a type $s$ and some $\varepsilon>0$ such that $\beta_{i}^{-1}(b)=\{s\}$ and $\beta_{j}^{-1}(b)=\{\underline{s}\}$ for every $b \in$ $(\underline{b}, \underline{b}+\varepsilon)$. Since $\pi_{i}\left(\underline{b}^{+}, s\right)=0$, we have that $\pi_{i}(b, s)=u(b, s, \underline{s}) Q_{i}(b, \underline{s}) f(\underline{s} \mid s)=0$ for every $b \in(\underline{b}, \underline{b}+\varepsilon)$. Thus, $u(b, s, \underline{s})=0$ for every $b \in(\underline{b}, \underline{b}+\varepsilon)$, a contradiction that establishes the claim.

Since both bidders' strategies are monotone at $\underline{b}$, the preceding claim and the proof of Lemma 10 imply that $\beta_{i}^{-1}(\underline{b})=\beta_{j}^{-1}(\underline{b})=\{\underline{s}\}$ and that $u(\underline{b}, \underline{s}, \underline{s})=0$. Thus, if the type $\underline{s}$ of bidder $j$ bids with positive probability in some interval $(\underline{b}, \underline{b}+\varepsilon)$, we have that $\pi_{i}(b, \underline{s})=u(b, \underline{s}, \underline{s}) Q_{i}(b, \underline{s}) f(\underline{s} \mid \underline{s})<0$ for $b \in(\underline{b}, \underline{b}+\varepsilon)$. We conclude that the type $\underline{s}$ of bidder $i$ bids $\underline{b}$ with probability 1 . Part (ii) follows.

Acknowledgements: I am especially grateful to Charles Wilson for innumerable discussions on this topic.

\section{References}

[1] R. Engelbrecht-Wiggans, P. Milgrom and R. Weber, Competitive bidding and proprietary information, Journal of Mathematical Economics 11 (1983), 161-169.

[2] J.Griesmer, R. Levitan and M. Shubik, Toward a study of bidding processes, Part IV: Games with unknown costs, Naval Research Logistics Quarterly 14 (1967), 415-433.

[3] K. Hendricks, R. Porter and C. Wilson, Auctions for oil and gas leases with an informed bidder and a random reservation price, Econometrica 62 (1994), 1415-1144.

[4] S. Karlin, "Total Positivity: Volume I", Stanford University Press (1968)

[5] S. Karlin AND Y. RinotT, Classes of orderings of measures and related correlation inequalities. I. Multivariate totally positive distributions, Journal of Multivariate Analysis 10 (1980), 467-498.

[6] E. Maskin And J. Riley, Optimal auctions with risk averse buyers, Econometrica 52 (1984), 1473-1518.

[7] E. Maskin And J. Riley, Existence and uniqueness of equilibrium in sealed high bid auctions, University of California, Los Angeles, working paper \# 407, March 1986. 
[8] E. Maskin And J. Riley, Equilibrium in sealed high bid auctions, Draft. December 1996.

[9] E. Maskin And J. Riley, Uniqueness in sealed high bid auctions, Draft. December 1996.

[10] P. Milgrom, Rational expectations, information acquisition and competitive bidding, Econometrica 50 (1982), 1089-1122.

[11] P. Milgrom and C. Shanon, Monotone comparative statics, Econometrica 62 (1994), 157-180.

[12] P. Milgrom And R. Weber, A theory of auctions and competitive bidding, Econometrica 50 (1982), 1081-1122.

[13] P. Milgrom and R. Weber, Distributional Strategies for games with incomplete information, Mathematics of Operations Research 10 (1985), 619632 .

[14] G. Rodriguez, "Essays in Auction Theory", Ph.D dissertation, New York University, 1994.

[15] W. Wнiтt, Multivariate monotone likelihood ratio and uniform conditional stochastic order, Journal of Applied Probability 19 (1982)

[16] R. Wilson, Strategic analysis of auctions, in "Handbook of Game Theory" (R. Aumann and S. Hart, Eds), North Holland, 1988. 